\title{
IRIS MURDOCH ON ART, ETHICS AND ATtention
}

\author{
Anil Gomes \\ Trinity College, University of Oxford \\ Forthcoming, The British Journal of Aesthetics [accepted 2013]
}

\begin{abstract}
Can the experience of great art play a role in our coming to understand the ethical framework of another person? In this paper I draw out three themes from Iris Murdoch's The Sovereignty of Good in order to show the role that communal attention to works of art can play in our ethical lives. I situate this role in the context of Murdoch's wider philosophical views.
\end{abstract}

\section{Introduction}

The three essays which make up Iris Murdoch's The Sovereignty of Good constitute an extended reflection on the nature of goodness and a sustained defence of the thought that great art can and should play a role in our ethical lives. ${ }^{1}$ My aim in this paper is to draw out some of the ideas and themes that support Murdoch's claims in these essays. I will suggest that Murdoch's discussion isolates one form in which the experience of art has ethical significance: a form which centres upon the communal nature of art appreciation.

\footnotetext{
${ }^{1}$ (Murdoch 1970). I will use the following abbreviations to refer to the individual essays: 'The Idea of Perfection' (IP), 'On 'God' and 'Good” (G) and 'The Sovereignty of Good over Other Concepts' (SG). All page numbers refer to the Routledge Classics edition (2001). I will refer to (Murdoch 1957) as (S\&G), to (Murdoch 1959) as (SBR) and to (Murdoch 1992) as (MGM).
} 
The relation between art and ethics is as much a topic of Murdoch's fiction as her philosophy, and her discussion of the subject spans an array of concerns. Central to this reflection is the question of whether art can make one a better person, and in providing an affirmative answer, Murdoch locates a role for aesthetic experience in our ethical lives comparable to that played by prayer in religious communities. Art, like prayer, draws us away from the self towards an appreciation of that which is good. In this way, great art makes one a better person.

The question I want to consider is in some ways more constrained: not whether attending to works of art can make an individual subject a morally better person, but whether such experience has any role to play in our coming to understand and share the ethical framework of another person. Murdoch writes:

Human beings are obscure to each other, in certain respects which are particularly relevant to morality, unless they are mutual objects of attention or have common objects of attention... (IP, p.32)

My question is whether works of art can serve as these common objects of attention: can the process of jointly attending to a work of art with another person make her moral framework less obscure? This is in part a question about the acquisition of certain concepts, since coming to share another's ethical framework may involve coming to possess various concepts involved in that framework. But it is primarily a question about understanding and the role that art might play in enabling me to comprehend another's ethical point of view.

I'll begin by drawing out three themes from Murdoch's essays: the thickness of aesthetic concepts; the interweaving of the ethical and aesthetic; and the perceptibility of the aesthetic. These three themes can be used, I'll suggest, to support the claim that the act of jointly attending to works of art can play a role in one's coming to understand and share another's ethical framework. In the final part of this paper I'll show how this links into wider themes in Murdoch's philosophy. 


\section{Three Themes}

\section{The Thickness of Aesthetic Concepts}

According to Murdoch, many value terms - which include both the ethical and aesthetic - have both normative and descriptive elements. In contemporary terminology, they express thick concepts. ${ }^{2}$ Discussions of thick concepts often focus upon the ethical: courageous, cruel, kind, treacherous, chaste and so forth. But it is easy to think of aesthetic examples: imaginative, witty, crude, inelegant, dainty and dumpy. The descriptive element of a thick concept entails that its application is world-guided: in applying the concept one is responsive to the non-evaluative properties of the object, and any such application can turn out to have been false if the non-evaluative facts were not as the judger took them to be. The normative element, in contrast, entails that the application is evaluative: in applying the concept one also evaluates the item in question as something to be criticised or praised. Application of a thick aesthetic concept both describes and evaluates the item so judged.

Part of the philosophical interest in thick concepts is that they seem to defy bifurcation into purely descriptive and evaluative components. ${ }^{3}$ Murdoch certainly rejects any such disentanglement: the value concepts, she says, are 'patently tied onto the world' (SG, p.88), to think otherwise is to divorce value claims from the world of factual propositions, a move which would involve the relegation of value to 'a shadowy existence in terms of emotive language, imperatives, behaviour, patterns, attitudes' (G, p.57). ${ }^{4}$ Part of her concern seems to be that the disentanglement of fact from value would entail that one could fix on all the facts whilst leaving it open whether the value component was applicable; that there could be a specification of the item which was neutral as to which evaluative attitude one should take towards it. For Murdoch, it is the world itself which

2 (Williams 1985: pp.129-30, pp.140-142)

${ }^{3}$ See, for example, (Williams 1985: pp.141-145), (McDowell 1983: esp. pp.201-203),

(Foot 1958-59: \$1) and (Putnam 2002: pp.28-31). For a dissenting opinion, see (Blackburn 1992).

${ }_{4}^{4}$ On Murdoch's rejection of the fact/ value distinction see (MGM, ch.2) and (Putnam 2002: p.38). 
determines which thick concept is appropriate, and in so doing it can fix the evaluative attitude we should hold towards it. ${ }^{5}$

This line of thought can perhaps be supported by John McDowell's observation that it may be only from a particular evaluative point of view that the descriptive elements common to an aesthetic kind form any unified class. ${ }^{6}$ Consider our use of the term 'elegant', as applied to music, novels, architecture, ways of walking, cuts of cloth and so on. It seems unlikely that all such things so described share any purely descriptive properties, yet we do not want to say that our use of the term varies from context to context. Rather, we should take it that the normative element is integral to the concept's descriptive content and that it may be a condition on the possession of an aesthetic concept that one understands the relevant evaluative point of view. ${ }^{7}$ It is the failure of bifurcation which supports the thickness of aesthetic concepts.

What is required for a subject to grasp a thick aesthetic concept? Given this entanglement, any subject who is to be credited with possession of a thick aesthetic concept must understand the concept's normative element. Without grasp of this normative component, she will not be able to fix the reference of the concept and, in particular, will not be able to continue applying the concept to new cases. Understanding the normative component involves understanding the way in which the application of the concept expresses a particular evaluative attitude towards the things so judged and appreciating how the expression of that evaluation is tied up with certain wider beliefs, values and concerns.

Note that understanding here does not imply endorsement: one can understand the normative component without endorsing its point of view. A.W. Moore's distinction between engaged and disengaged ways of grasping a thick concept makes this clear. ${ }^{8}$ To grasp a thick concept in the disengaged way is to be able to recognise when applications of the concept would be appropriate, to understand others' application of it and so on. To

\footnotetext{
${ }^{5}$ Murdoch's rejection of bifurcation thus involves a robust realism about the referents of evaluative concepts. See (McDowell 1983) and (Price 2000) for discussion of the relation between thickness and realism.

${ }^{6}$ (McDowell 1983: pp.201-203)

7 Williams has pushed this thought most forcefully. See (Williams 1985: pp.141-145). Williams says that he first heard this 'Wittgensteinian idea... expressed by Philippa Foot and Iris Murdoch in a seminar in the 1950s' (Williams 1985: p.240, fn.7).

8 (Moore 2006: p.137). Moore notes that the distinction is found in Williams's original discussion.
} 
grasp a concept in the engaged way is 'not only to be able to do these things, but also to feel sufficiently at home with the concept to be prepared to apply it oneself... What this requires, roughly, is sharing whatever beliefs, concerns, and values give application of the concept its point.' (Moore 2006: p.137). On both ways of grasping the concept one must understand the beliefs, concerns and values which give application of the concept its point, but it is only in order to possess the concept in the engaged way that one must endorse those values as one's own.

Moore uses the concept of the Sabbath to illustrate his distinction: 'Those who are not Jewish have no difficulty in grasping this concept in the disengaged way. A person who is not Jewish can understand perfectly well what someone means when she says that her birthday this year falls on the Sabbath. But only a Jewish person recognizing an obligation to keep the Sabbath can grasp the concept in the engaged way. We might say that such a person lives by the concept' (Moore 2006: p.137). Similar cases arise in the aesthetical realm.

Consider the musical term 'lo-fi'. When used in an aesthetic judgement, this term does more than simply describe poor-quality sound recordings: it conveys an evaluative stance towards a certain way of recording and presenting music, one which rejects mainstream recording techniques as dishonest and illusory. Someone who possesses this concept in the disengaged way is able to make use of the concept: she can understand those who use the concept and can apply the concept herself. But she may be able to do this whilst rejecting what she takes to be the inverse snobbery which underlies the concept's use. Or consider Jane Austen's use of the term 'elegant'. Elegance involves, for Austen, a sense of refinement and choice adornment; it requires gentility; it enhances beauty; it relates to delicacy. ${ }^{9}$ A reader of Austen may grasp the concept sufficiently to understand her applications whilst dissenting from the beliefs and values which make difficult the instantiation of elegance by men. ${ }^{10}$ Those who live by these concepts possess them in an engaged way.

To summarise: thick aesthetic concepts have both descriptive and evaluative components. The evaluative component comprises the beliefs, concerns and values which give application of the concept its point. Grasp

${ }^{9}$ These examples are taken from (Phillips 1970: pp.51-53); see the references therein. Ryle discusses Austen insightfully in (Ryle 1968)

10 (Phillips 1970: p.53) 
of an aesthetic concept requires that one understand these beliefs, concerns and values though it does not require that one endorse them for endorsement is only required to grasp the concept in an engaged way. Let me call these beliefs, concerns and values the evaluative frame of the thick concept in question. We can think of them as constituting the aesthetic point of view from which application of the concept makes sense. In order to understand how the term is to be applied, one has to grasp the concept's evaluative frame. I take this to capture Murdoch's insistence on the thickness of aesthetic concepts.

\section{The Interweaving of the Ethical and Aesthetic}

The second theme I want to draw out concerns the relation between the ethical and aesthetic. 'Goodness and beauty' Murdoch says 'are not to be contrasted but are largely part of the same structure' (IP, p.40). For 'aesthetic situations are not so much analogies of morals as cases of morals. Virtue is au fond the same in the artist as in the good man...' (IP, p.40; also G, p.58). ${ }^{11}$ How should we understand these claims?

An initial place to start thinking about the relation between these two domains is the facility with which we apply paradigmatically ethical concepts within the aesthetic domain. A painting can be honest, a novel can be brave and a piece of music may display integrity. Correlatively, some of our terms of ethical evaluation have distinct aesthetic overtones: people and actions can be fine, pure, rotten, or tarnished. ${ }^{12}$ Such an interweaving of ethical and aesthetic terms is a feature of our moral and artistic practice, and Murdoch takes this as evidence for these terms being part of the same structure. 'The good artist, in relation to his art, is brave, truthful, patient, humble' (SG, p.84), and the same can be said of the work he produces. The interweaving of ethical and aesthetic language is claimed to reflect some deeper interweaving of the values themselves.

One way to make sense of this idea is to take seriously the thought that the appreciation and production of art involves the exercise of genuine

\footnotetext{
${ }^{11}$ Cf. Wittgenstein: 'Ethics and aesthetics are one and the same' (TLP, 6.421); Murdoch: 'art and morals are, with certain provisos... one. Their essence is the same.' (S\&G, p.215) ${ }^{12}$ The examples come from (McGinn 1999: pp.92-93).
} 
virtues. ${ }^{13}$ This is something which Murdoch stresses throughout her work (SBR, p.284f; IP, p.40f; SG, p.84). The dispositions involved in artistic practice are classed as virtues in part because their exercise takes place 'under the concept of art', but also because they are partly constitutive of human well-being. ${ }^{14}$ If artistic practice involves genuine virtues, then we can give content to Murdoch's thoughts about art and ethics being part of the same structure (IP, p.40; S\&G, p.215): evaluations in both domains involve sensitivity to the virtues displayed by the relevant objects of approval.

But what makes these virtues part of the same structure? Murdoch's motivating thought is that our use of one set of terms in the other sphere is an extension without distortion or metaphor. ${ }^{15}$ Peter Goldie has argued that this is evidenced by the fact that some of these terms demand a degree of cross-situational consistency in order for application of the concept to be appropriate: 'if someone is an honest person, we would expect him to be honest in his intellectual or artistic activity as well as in his ethical dealings with other people' (Goldie 2008: p.189). Similarly, Gilbert Ryle notes how Jane Austen's characters show 'a prevailing correlation between sense of duty, sense of propriety and aesthetic taste' (Ryle 1968: p.297). If our use of terms in one sphere is connected to our use of them in the other, these connections tell against any attempted cleaving of the aesthetic and ethical virtues and in support of Murdoch's identification of one evaluative structure.

To try and give content to this claim, let me return to the notion of an evaluative frame: those beliefs, concerns and values that give application of a thick concept its point. Often a set of thick concepts will share enough of the beliefs, concerns and values that give meaning to their members that we can talk about the set's evaluative framework: the beliefs, concerns and values that comprise the evaluative element of a set of concepts. Consider a particular set of thick religious concepts, for example the set of Christian concepts which includes such examples as blasphemy, chastity, original sin, divine revelation and so forth. The evaluative frames of each of these concepts may overlap to such an extent that we can talk about the wider Christian evaluative framework - the beliefs, concerns and values which

13 (Goldie 2007), (Goldie 2008).

${ }^{14}$ See (Goldie 2008, §3), from whom I take the term 'interweaving'.

15 Thanks to a referee for this way of putting the point and for very helpful comments on what follows. 
structure the use of this set of concepts. The individuation conditions for such frameworks are not strict: whether a set of frames shows enough coherence and overlap that we are justified in talking about a wider evaluative framework may depend on the use to which we wish to put the idea. But I take it that there is something intuitive about this notion.

It is plausible that certain aesthetic concepts share an evaluative framework: it is the same beliefs, concerns and values which structure the use of elegance, delicacy, wit, imagination and creativity for example. The same is true for certain ethical concepts: honesty, integrity, courage and sincerity, say, share an evaluative framework. Murdoch's claim that art and morality are part of the same structure can be expressed using this notation as the claim that the evaluative framework of our aesthetic concepts overlaps with the evaluative framework of our ethical concepts. On this picture, the difference between aesthetic and ethical values is one of degree and not kind: aesthetic and ethical values lie on a spectrum.

Note that this is not simply a claim about the difficulties of limning the aesthetic, though that, of course, follows. Rather, I take Murdoch's thought to be that talk of two distinct domains is misleading: there is only one evaluative domain in which the aesthetic and ethical overlap. The point of view from which one makes sense of aesthetic activities and the point of view from which one makes sense of ethical activities are not distinct. No doubt there are important differences between our aesthetic and ethical practices but these do not reflect any deep difference in kind between the values themselves. ${ }^{16}$

To say this is not to defend the claim: my concern for the moment is simply to clarify what Murdoch might mean by talk of art and ethics being part of the same structure. (I discuss criticisms of the claim in $\$ 4$ below.) I take the idea of overlapping evaluative frameworks as being capable of explaining the facility with which we use aesthetic and ethical terms across both domains and as justifying Murdoch's characterisation of art as involving virtues. That an artist and artwork can be brave in the same way that a fireman can be brave - or even that a person can be fine in the same way that a play can be - is a result of both evaluations drawing upon the

\footnotetext{
${ }^{16}$ See also (G, pp.55-57) on the unitary nature of value; (SG, pp.84-85) on the relation of aesthetic and ethical virtues; and (SG, p.96ff.).
} 
same beliefs, concerns and values. Aesthetics and ethics are part of the same structure because they involve overlapping evaluative frameworks.

\section{The Perceptibility of the Aesthetic}

The final theme I want to consider concerns the perceptibility of the aesthetic. That there are thick aesthetic concepts does not yet show that the features such concepts pick out are perceptible. Chastity, for example, is a thick ethical concept, but one cannot tell by looking whether or not someone is chaste. But beauty can be a matter of the senses (G, p.58), and we think of our application of certain aesthetic concepts as responsive to how things look. For Murdoch, '[b]eauty is the convenient and traditional name of something which... gives a fairly clear sense to the idea of quality of experience [and] change of consciousness.' (SG, p.82; see also MGM, p.3). ${ }^{17}$ This is to say: certain aesthetic qualities of a work of art contribute towards the character of our experience. Some aesthetic properties are perceptible.

This contrasts, Murdoch suggests, with the ethical: it is 'as if we can see beauty itself in a way in which we cannot see goodness itself... I can experience the transcendence of the beautiful but not (I think) the transcendence of the good.' (G, p.58). Murdoch hesitates slightly in drawing this contrast, but this hesitancy is not to be explained by her focus in this sentence on the 'thinner' notions of beauty and goodness; the contrast holds, she suggests, 'even when we see the unselfish man in the concentration camp' (G, p.59). Certainly there is something to the contrast: we think of an object's beauty as often contributing to the way it looks, whereas ethical properties do not seem as closely tied to appearances. Perhaps the fairest way to express this contrast, acknowledging Murdoch's slight pause, is to say that the aesthetic features of a work of art are sometimes perceptible in a way that that ethical features of a person or action are not. This draws the contrast whilst leaving it open whether goodness might be perceptible in some other sense. ${ }^{18}$

${ }^{17}$ The Routledge Classics edition (2001) has the typo 'arid' for 'and'.

${ }^{18}$ For a perceptual account of moral epistemology, which draws on Murdoch's work in these essays, see (McDowell 1979). 
How might one defend the claim that some aesthetic properties are perceptible? The question of how to determine which properties feature in our experience is a vexed one in the philosophy of perception, and one might worry that all Murdoch offers in defence is an appeal to intuition. ${ }^{19}$ Nevertheless, the idea that we perceive elegance, wit, daintiness and dumpiness is not especially contrary to common sense, and Murdoch has at least the support of the etymology of the term 'aesthetic'. Perhaps the strongest consideration in favour concerns our capacity to recognise these aesthetic features. When we identify such features we typically recognise them on the basis of our experience of them: it is by hearing the music that we identify it as elegant. And our sensitivity to these aesthetic features can make a difference to the character of the experience: as one develops the capacity to recognise elegance one comes to hear the music as elegant. What explains the phenomenological difference made by this recognitional capacity? The best answer seems to be that in such cases the aesthetic attributes genuinely feature in one's experience. ${ }^{20}$ Such an appeal to phenomenology would support the claim that some aesthetic properties are perceptible.

Note that this defence relies on the thought that the experiences of those who are sensitive to these aesthetic features differ in phenomenological character from those who are not so sensitive: it is because I am capable of recognising elegance that I hear music as elegant. What is involved in possessing this recognitional capacity? Recognition implies some sort of standing capacity to identify things as elegant. And if elegance is a thick aesthetic concept, then subjects can only recognise elegance given some understanding of the evaluative point of view from which the items judged to be elegant form a unified class. Without some grasp of the concept's evaluative frame, a subject would not be able recognise items as displaying elegance. This suggests that although some aesthetic features are perceptible, they are not manifest: such features are only perceptible to one who grasps the aesthetic point of view from which it is possible to recognise the feature in question. ${ }^{21}$

\footnotetext{
${ }^{19}$ For recent commentary on the question of which properties feature in the contents of experience, see the papers in (Hawley and Macpherson 2011).

20 Susanna Siegel makes an argument along these lines in support of the claim that natural kind properties feature in the contents of one's visual experience. See (Siegel 2007).

${ }^{21}$ Does this perceptibility require an engaged grasp of the concept? No: engaged and disengaged possession both enable a subject to recognise instantiation of the aesthetic feature for one can take up an aesthetic point of view without endorsing the belief,
} 
We can bring this out by considering the contrast between the experiences of a novice who lacks a particular aesthetic concept and those of an expert who possesses it. ${ }^{22}$ Consider two people attending to a piece of music, one of whom grasps the concept of elegance and one of whom lacks it. It seems plausible that the phenomenal character of their experiences will differ: the subject who possesses the concept of elegance has an experience which represents the music as elegant. The best explanation of this, I have suggested, is that elegance is a genuinely perceptible quality of the music. But it is only perceptible to the person who has a grasp of the concept of elegance: it is because the expert is capable of recognising things as elegant that the music seems to her to be elegant. And, given the requirements on possessing the concept of elegance, this means that it is only perceptible to one who understands the evaluative component of the concept. Perceiving elegance require the capacity to recognise things as elegant and this, in turn, requires an understanding of the evaluative point of view which groups together things as elegant.

This fits with our intuitions about the perceptibility of these aesthetic qualities. Although it is natural to say that we can hear the elegance in a piece of music, it is not hard to imagine well-intentioned observers with fully functioning sensory apparatuses who were insensitive to this aesthetic feature. While the elegance is perceptible to those of us who are capable of recognising the feature, it is not perceptible to those who do not understand the relevant evaluative frame. Perceiving an aesthetic quality requires sense and sensibility. This is the final theme I want to draw from Murdoch.

In the next section I will suggest that we can use these three claims - the thickness of aesthetic concepts, the interweaving of the ethic and aesthetic and the perceptibility of some aesthetic qualities - to construct a framework to explain why joint attention to works of art can play a role in a subject coming to understand another person's ethical viewpoint. To the extent that this framework relies on each of these claims, it will inherit any concerns one might have about the three elements. Nevertheless what I hope to show is that there is reason for one who is sympathetic to these claims to accord the communal experience of attending to works of art a

concerns and values which constitute that view. And so long as a subject is capable of recognising the aesthetic feature, there are grounds for holding that the presence of the feature makes a difference to the phenomenal character of the experience.

22 This is how Siegel motivates her view: (Siegel 2007). 
central role in explaining our ability to understand another's ethical points of view.

\section{Joint Attention}

We can begin consideration of this issue by thinking about the role that aesthetic experience plays in our grasp of thick aesthetic concepts. There seems good reason to accord experience a role in explaining our grasp of some of these concepts: aesthetic concepts are not, after all, purely theoretical concepts whose meaning derives solely from their inferential relations to other propositions in a structured theory. Rather, some of them are partly experiential in the sense that facility in the use of such concepts would seem to presuppose acquaintance with the aesthetic feature in question. Someone who had never experienced a delicate object would appear to have a diminished conception of the term. So it seems that experience should play a role in explaining our grasp of certain aesthetic concepts.

But there is a prima facie difficulty with seeing how this is possible given the first and third of the themes discussed above. According to the perceptibility of the aesthetic, those aesthetic properties of an artwork which are perceptible are so only to those who already have some standing capacity to recognise and identify the aesthetic feature in question. But, given the thickness of aesthetic concepts, this capacity for identification over time itself requires an understanding of the evaluative point of view from which the items judged form a unified class. Without such an understanding, there would be no discernable shape for the subject to recognise. So far from being capable of explaining our grasp of an aesthetic concept, the perception of these aesthetic properties seems already to presuppose an understanding of the evaluative point of view involved in grasping the concept. The explanans presupposes what was to be explained.

One can get a feel for the difficulty here by contrasting two extreme forms of inadequate explanation. Imagine first a subject simply staring at a work of art from an alien aesthetic culture. There seems no way for her to use such experience to ground her grasp of the aesthetic concepts used by that culture in evaluating the work of art. For the aesthetic features which that culture finds in the artwork are inaccessible to the perceiving subject without a prior understanding of the alien culture's evaluative framework. And contrast this with a subject who aims to ground her grasp of an 
aesthetic concept without any experience of the aesthetic feature in question: someone who tries to understand the aesthetic concepts of an alien culture purely by reading the reviews of their well-informed critics. Such a person might get some idea of the ways in which various of the alien aesthetic terms relate to each other, but her understanding would be deficient in an important way as a result of her never having experienced the aesthetic features in question.

One response to the inadequacy of these suggestions would be to drop the idea that aesthetic experience need play a role in explaining our grasp of aesthetic concepts and allow that one could come to understand the aesthetic concepts of an alien culture without any experience of the properties in question. But Murdoch would not countenance such a response: learning, she says, takes place in the context of particular acts of attention (IP, p.31). Instead she offers us a middle course:

\begin{abstract}
Words... have both spatio-temporal and conceptual contexts. We learn through attending to contexts, vocabulary develops through close attention to objects, and we can only understand others if we can to some extent share their contexts... Uses of words by persons grouped round a common object is a central and vital human activity. The art critic can help us if we are in the presence of the same object and if we know something about his scheme of concepts. (IP, p.31)
\end{abstract}

Experience of an artwork can ground our grasp of an aesthetic concept when we jointly attend to it in the presence of an informed other. Joint attention explains our grasp of aesthetic concepts.

Some familiar examples illustrate the plausibility of Murdoch's suggestion. Consider the jazz enthusiast who helps another appreciate the aesthetic qualities of John Coltrane's Ascension by drawing her attention to patterns in what she first hears as noise. Or the guided tours in art galleries in which a curator talks to school children about a painting and introduces them to some aesthetic terminology and ideas. In each of these cases, a subject comes to understand an aesthetic concept through attending to a work of art in the company of a more informed other.

A more striking case comes from a recent paper by Greg Currie. ${ }^{23}$ His topic is the aesthetic status of cattle amongst the cattle breeding Dinka

${ }^{23}$ (Currie 2011). See also (Coote 1994) and (Ryle 1982). 
people. In defence of the claim that such cattle are works of art, Currie points to the fact that the Dinka intentionally manipulate the appearance of cattle with what appear to be aesthetic results. The resulting qualities are picked out with a specific critical vocabulary:

[w] hen discussing the colour pattern of an animal-as they do for hours-the Dinka sound more like art critics than stockbreeders. For instance, when does mathiang — dark brown-become malual_reddish brown? If the animal has brown patches, are they large enough to make it mading or are they the smaller mottling that identifies malek? (Ryle 1982, quoted in Currie 2011: p.108).

This critical vocabulary seems alien to us. What would be needed to come to share the Dinka aesthetic conceptual scheme? We can't appreciate this critical vocabulary, Currie claims, 'without the training provided by substantial acquaintance with the works concerned, substantial knowledge of the techniques involved, and a good deal of insight into the broader role of these activities in the societies that nurture them' (Currie 2011: p.111). Joint attention provides a situation in which these conditions are fulfilled: by attending to the Dinka cattle in the presence of someone who knows the culture, we can come to understand their aesthetic concepts.

Note that Murdoch does not present such episodes of joint attention as a necessary condition on the possession of an aesthetic concept: the claim is only that joint attention is a central part of our standard grasp of these concepts. And it is the dual nature of joint attention which allows it to play this role: when we engage in an episode of joint attention, we are presented both with the artwork itself and a co-attender whose beliefs, concerns and values identify some aesthetic feature of the work. 'Progress in understanding a scheme of concepts often takes place as we listen to normative-descriptive talk in the presence of a common object' (IP, p.31). Hence the importance of joint attention in grounding our understanding of the aesthetic. ${ }^{24}$

Given the second of Murdoch's three themes, it is now but a short step to the claim that joint attention to a work of art can help us in understanding another's ethical framework. For grasping an aesthetic concept requires us to grasp the evaluative frame which structures its use; that is, the beliefs, concerns and values which give application of the concept its point. And

24 (Roessler 2005) draws attention to the role of joint attention in Murdoch's discussion. 
the beliefs, concerns and values which structure a subject's aesthetic concepts overlap with the beliefs, concerns and values which structure her ethical concepts. So a subject who comes to understand an aesthetic conceptual scheme has partial entry into the evaluative point of view from which ethical qualities are identified. Thus if joint attention can play a role in our coming to understand the evaluative framework of another's aesthetic concepts, it can put us on the road to understanding the evaluative framework of another's ethical concepts.

To see how this might work, consider again the Dinka. Their notion of dheeng, translated most often as 'dignity' (Deng 1972: p.14), appears to have a strong ethical dimension: '[a]s a noun, it means nobility, beauty, handsomeness, elegance, charm, grace, gentleness, hospitality, generosity, good manners, discretion, and kindness' (Deng 1972, quoted in Coote 1992: p.264). But there is also a perceptual dimension to the concept: people who are adheng (the adjectival form) are expected to exhibit various perceptible qualities. And many of these perceptible qualities relate to the aesthetic qualities that the Dinka find in cattle (Coote 1993: p.264). Accept for the sake of argument that the concept of dheeng and the concepts of mathiang, malual, mading and malek are part of the same evaluative structure. Then if joint attention with an informed other can enable one to grasp the aesthetic concepts of mathiang, malual, mading and malek, it can thereby make the partly ethical concept of dheeng less obscure. Understanding the Dinka aesthetics helps us some way towards understanding their ethics.

Note that there is no expectation in Murdoch that this process will be immediate or easy: all sorts of difficulties may beset us when we move from understanding another's aesthetics to understanding their ethics. The point is only that entry into another's aesthetic conceptual scheme makes such ethical understanding possible: in coming to understand another's art, one becomes disposed to understand her ethics. This is the force of talking about one evaluative domain in which both aesthetic and ethical values inhere. Art is a case of morals (IP, p.40) and in learning about another's art we are brought into the point of view from which her ethical discriminations are also made. 
Note also that the kind of interaction suggested by this account can and does take a variety of forms. ${ }^{25}$ Episodes of joint attention involve the subjects opening themselves up to each other's normative sensibilities and it is this process of co-ordination which allows them to develop an aesthetic and ethical understanding. In the case of the art critic and student, there is an asymmetry between those attending: the novice opens herself up to the expert in order to be guided and informed by her knowledgeable take on the object without any expectation that the expert will be similarly attuned to the student's reactions. But there are many cases in which there is no such imbalance: both of us are competent concept users with different frames of reference and the process of our jointly attending allows us to become sensitive to each other's sensibilities. Such variety illustrates the multiplicity of ways in which communal acts of aesthetic attention can enable interpersonal aesthetic and ethical understanding: mutual influence and understanding can take many forms. ${ }^{26}$

It is this potential for locking onto one another's normative sensibilities which explains why Murdoch should accord the communal experience of a work of art a prominent place in the explanation of how it is that we can come to understand another's ethical framework. An episode of joint attention presents us both with the artwork itself and a co-attending subject for whom the aesthetic features of the artwork are perceptible. Jointly attending to the work of art together with this person enables me to perceive those aesthetic features through a gradual understanding of the aesthetic point of view which picks them out. Understanding such a point of view involves understanding the beliefs, concerns and values that structure the co-attender's use of her aesthetic concepts - and these beliefs, concerns and values are equally at play in her ethical framework. Thus in coming to understand her aesthetic point of view I put myself in a position to understand her ethical point of view. Joint attention to works of art can help to make another's ethical life less obscure.

25 Murdoch is sensitive to such variety: see (MGM, chs.1 and 4).

26 The account offered here concerns itself solely with the issue of how joint attention can enable interpersonal ethical understanding between those involved in the communal act of attention. It is a further and distinct question whether and how such attention can play a role in our coming to understand the artist's ethical framework. 


\section{Unity}

What should we say about this framework? The idea that joint attention might play a role in explaining our grasp of aesthetic concepts is, I think, a plausible one: it is true, as Murdoch says, that the art critic can help us if we are in the presence of the same object, and that this shared context is relevant to 'seeing more' to 'seeing what she sees' (IP, p.31). But extending this to the case of ethics seems more problematic. In particular, a great deal of weight is placed on the second of Murdoch's themes: the claim that aesthetics and ethics are part of the same structure. It is only if one accepts this claim about the relation between art and ethics that the proposed extension can take place. And this may cause consternation in those minded to deny the claim.

Consider one source of resistance to the claim that aesthetic and ethical concepts are part of the same evaluative structure, namely a concern about the demarcation of the respective domains. Grant that aesthetic and ethical concepts are part of the same evaluative structure. Then someone who grasps the evaluative framework of an aesthetic concept has part entry into the evaluative point of view from which ethical qualities are identified. But, as Peter Goldie notes, 'we know perfectly well that good people can be blind to art; and we know too that bad or profoundly selfish people can be great artists, or critics of superb judgement and taste.' (Goldie 2007: p.384). If Murdoch's claim is correct, a subject who grasps an aesthetic concept thereby grasps some of the beliefs, concerns and values which comprise the evaluative framework of ethical concepts. Yet the profoundly selfish artist seems no closer to understanding the ethical point of view than someone who lacks the capacity to make any aesthetic discriminations. How are we to explain the possibility of great artists who lack moral virtues if possession of an aesthetic concept entails partial grasp of the ethical point of view? Goldie's selfish artist looks like a counterexample to the claim that aesthetic and ethical concepts are part of the same evaluative structure.

This concern is particularly pressing given the presence of an alternative line of thought in Murdoch's writings, one which locates a link between art and ethics in terms of the attitude one stands in towards the objects of aesthetic and ethical evaluation rather than in the aesthetic and ethical concepts themselves. The process of appreciating great art, Murdoch suggests, can lead to moral improvement through the inculcation of a 
capacity for attention. ${ }^{27}$ Learning to appreciate a work of art involves attending closely to its features; it involves the ability to make fine discriminations; it requires putting to one side any personal prejudices; it calls for a 'just and loving gaze directed upon an individual reality' (IP, p.33). These skills are drawn upon equally in ethical contexts: in determining whether an action is courageous or foolhardy, we are required to attend closely to its features, make fine discriminations and see the act for what it really is. In this way, the experience of art can be an exercise in moral education: it develops in the subject a capacity for looking at things in a certain way - a capacity which has an important analogue in the sphere of moral epistemology.

It is this ethical context which features at the start of Murdoch's Metaphysics as a Guide to Morals:

The idea of attention or contemplation, of looking carefully at something and holding it before the mind, may be conveyed early on in childhood. 'Look, listen, isn't that pretty, isn't that nice?'. Also, 'Don't touch!'. This is moral training as well as preparation for a pleasurable life. (MGM, p.3)

Here the thought is that attention to aesthetic objects aids moral understanding through the development of a way of appreciating the objects of evaluative concern. 'Children, if they are lucky, are invited to attend to pictures or objects, or listen quietly to music or stories and verses' (MGM, p.3), and this process of attending to art supports a pattern of behaviour which has application in ethical contexts.

This link between art and ethics in terms of the attitude one takes towards the objects of evaluation is more restrained than the shared evaluative framework discussed above: it claims only that both domains involve a certain sort of receptivity, and that the skills and capacities involved in such receptivity can be developed through the activities involved in art appreciation. Murdoch terms such a process 'unselfing': the experience of art helps me to develop the capacity to go beyond the personal prejudices arising from my own ego and see instead things as they really are (G, p.63, pp.68-69). But it is important to note there is nothing distinctive about art in this regard: religion (G, pp.53-54), love (MGM, pp.16-17), nature (SG,

${ }^{27}$ Murdoch takes the notion of attention and its correlates from her reading of Simon Weil. See, in particular, the section on 'Attention and Will' in (Weil 2005). 
p.82), history, chemistry (SG, p.88), even the learning of another language (SG, p.87) can all play a role in developing such a capacity. So there is reason to think that this process of 'unselfing' - as central as it is to Murdoch's philosophy - cannot capture all that she takes to be distinctive about the relation between art and ethics. It is the stronger claim that ethics and aesthetics are part of the same structure which makes good Murdoch's insistence that aesthetic and ethical value should be in some sense unitary' (G, p.55-56).

Given the importance of this claim in motivating the thought about joint attention, I want to end by situating it in Murdoch's wider philosophical framework. In particular, I want to draw out its connection to a deep and pervasive thread in her philosophical views. For lying behind the claim that the ethical and aesthetic share an evaluative domain is a particular belief in a certain sort of philosophical unity. 'It is the traditional inspiration of the philosopher, but also his traditional vice, to believe that all is one', Murdoch writes, later adding - as if there were any doubt - that her 'own temperament tends to monism' (G, p.49). It is this temperament which is evidenced in Murdoch's claims about the relation between art and ethics.

Consider again the objection of the selfish artist. One response to this possibility would be to retreat to the account of 'unselfing' mentioned above. On this proposal, the claim about ethics and aesthetics sharing a common structure is dropped in favour of a link in terms of the attitude one takes towards the objects in each domain. Using the terminology of late nineties higher-education, we might call this a transferable skills account of art appreciation for it holds that aesthetic appreciation involves exercise of the same skills and capacities deployed in the moral realm. Joint attention may remain important on this picture, not because it enables one to clue in to the aesthetic features to which one's co-attender is sensitive, but because it provides a context in which one can learn what it is to patiently and lovingly attend to an object of normative evaluation (MGM, p.3).

I objected above that this proposal weakens the connection between art and ethics since Murdoch allows that many other forms of activity can play a similar 'unselfing' role. But a defender of this proposal might counter that the experience of great art militates against selfishness in a particularly powerful way: it 'invigorates our best faculties and... inspires love in the highest part of the soul' (SG, p.83). This is one way to read 
Murdoch's claim that although intellectual disciplines such as mathematics, history and chemistry can play 'the same kind of role as that which I have attributed to art' (SG, p.87), they are only 'introductory images of the spiritual life [and not] the spiritual life itself (SG, p.88). It is the experience of art which most clearly enables one to develop the kind of 'judicious respectful sensibility' (SG, p.87) required for ethical deliberation. Whether or not this is plausible depends on the extent to which the experience of great art is genuinely exceptional when compared with other 'unselfing' activities.

A second, stronger, response would be to delimit the interweaving claim. Goldie's example of the selfish artist looks to challenge the claim that grasp of the evaluative framework of an aesthetic concept suffices for grasping at least some of the beliefs, concerns and values which constitute the evaluative framework of certain ethical concepts. But one could accept that this claim fails to hold with full generality whilst insisting that certain works of art are such that grasp of their aesthetic properties puts one in a position to grasp certain ethical concepts. On this way of avoiding the problem, the second of Murdoch's themes is not rejected but circumscribed: it is only in certain works of art that the aesthetic and ethical are inseparably intertwined and joint attention to those artworks can enable ethical understanding.

Murdoch suggests that religious art in the Christian tradition may involve such entanglement (MGM, ch.4). 'Christianity... is itself like a work of art' and 'the great painters, with their impressive, memorable, authoritative works, helped to create the unified pictorial conglomerate with which we are so familiar' (MGM, p.82). For these paintings, understanding their aesthetic properties requires understanding the religious concepts that they mean to convey for ' $r$ r] eligious imagery colours and fixes and bodies forth moral ideas.' (MGM, p.82). The Dinka aesthetic concepts provide another example. Perhaps aesthetic and ethical components are so bound together in the notion of dheeng that grasp of the Dinka aesthetic concepts thereby suffices for grasping at least part of the evaluative component of dheeng. In these specific cases, grasp of certain aesthetic concepts suffices for understanding some of the beliefs, concerns and values that make up the evaluative component of an ethical concept and, given such interweaving, joint attention can play a role in ethical understanding in this restricted domain. 
Each of these responses involves abandoning the claim that ethics and aesthetics are part of the same structure. Murdoch's own response is less acquiescent. Consider her discussion of the diversity of the virtues within the ethical domain.

[I] $\mathrm{f}$ we reflect upon courage and ask why we think it to be a virtue, what kind of courage is the highest, what distinguishes courage from rashness, ferocity, self-assertion, and so on, we are bound, in our explanation, to use the names of other virtues. The best kind of courage... is steadfast, calm, temperate, intelligent, loving... (G, p.56)

The thought here seems to be that when we reflect on the ethical virtues, we see that they involve a certain concordance: acting courageously involves acting kindly, lovingly, calmly and so on. So a person who is capable of acting courageously must also be capable of acting in accordance with these other virtues. The conditions on courageous actions show how the ethical virtues fit together: 'reflection rightly tends to unify the moral world, and... increasing moral sophistication reveals increasing unity.' (G, p.56). ${ }^{28}$

How does this relate to questions about the diversity of virtues across the ethical and aesthetic domains? Murdoch's suggestion is that this tendency towards unity holds not only within the ethical domain but also across the wider domain of the valuable: there is a concordance of the values and virtues involved across both ethical and aesthetic domains. ${ }^{29}$ Good art presents us with 'a truthful image of the human condition', it 'transcends selfish and obsessive limitations of personality' and, most of all, it exhibits a 'clear realistic vision with compassion' (SG, pp.84-5). In this way the production and appreciation of great art requires one to become a truthful, unselfish and compassionate person. The tendency towards unification we find in the ethical extends to embrace the aesthetic.

This comes out most strikingly in Murdoch's discussion of Plato's use of the sun as metaphor for the Form of the Good (SG, p.90f; G, p.68). The Good - like the sun - gives light and energy and enables us to know truth: 'In its light we see the things of the world in their true relationship.' (SG, p.90). Among the things it illuminates are both people and works of art: we speak 'perfectly seriously of ordinary things, people, works of art, as

${ }^{28}$ A similar point can be made about the aesthetic virtues: see (Gomes 2009).

${ }^{29}$ (G, p. 55), (MGM, ch.1) 
being good' (SG, p.90) for both stand under its illumination. Metaphors are modes of understanding (SG, p.91) and the suggestion is that both ethics and aesthetics are part of a shared domain, one structured by use of the concept 'good'. By reflecting on the way in which our ethical and aesthetic terms interrelate we can come to recognise the unity of our evaluative framework: 'moral advance carries with it intuitions of unity which are increasingly less misleading' (SG, p.93).

What about Goldie's counter-examples? Murdoch accepts that the connection between virtues cannot be all-encompassing: we must acknowledge that we are 'specialised creatures and merit in one area does not seem to guarantee merit in another' (SG, p.94). But when she considers examples analogous to Goldie's cases of good artists with ethical flaws or wicked people with sensitive tastes, she cannot bring herself to find balkanisation:

The good artist is not necessarily wise at home, and the concentration guard can be a kindly father. At least this can seem to be so, though I would feel that the artist had at least got a starting-point and that on closer inspection the concentration camp guard might prove to have his limitations as a father. (SG, p.94).

Murdoch's interweaving of the ethical and aesthetic struggles to find room for the troubling cases averred to in the quotation from Goldie above. For as long as the tendency towards unification is real, the proposed counterexamples can always be examined, and qualified, in this light.

Murdoch's inclination towards monism, then, - her 'unavoidable sense of unity' (G, p.57) - underlies the radical nature of these claims about the relation between art and ethics. The claim that aesthetic and ethical concepts are part of the same evaluative structure is sustained by a certain conception of philosophical unity: a unity which Murdoch finds supported by reflection but is largely an assumption of her wider framework. At the start of Metaphysics as a Guide to Morals, Murdoch writes, 'The urge to prove that where we intuit unity there really is unity is a deep emotional move to philosophy, to art, to thinking itself (MGM, p.1). It is this 'deep emotional move' which prompts Murdoch's interweaving of the ethical and aesthetical domains. And it is this interweaving which supports the thought that joint attention to great art can play a role in our coming to understand the ethical framework of 
another person. 'Human beings are obscure to each other, in certain respects which are particularly relevant to morality, unless they... have common objects of attention' (IP, p.32). We can find in Murdoch reason to hold that communal attention to works of art can help us gain insight into another person's ethical framework. But it is reason born of her temperamental urge towards philosophical unity. ${ }^{30}$

\section{References}

Blackburn, S. (1992) 'Morality and Thick Concepts', Proceedings of the Aristotelian Society, Supp. Vol. LXVI: 285-299.

Coote, J. (1992) 'Marvels of Everyday Vision: The Anthropology of Aesthetics and the Cattle Keeping Nilotes', in J. Coote and A. Shelton (eds.) Anthropology, Art, and Aesthetics Oxford: Oxford University Press: 245-275

Currie, G. (2011) 'Art and the Anthropologists', in A. Shimamura and S. Palmer (eds.) Aesthetic Science: Connecting Minds, Brains, and Experience. Oxford: Oxford University Press: 107-128

Deng, F. (1972). The Dinka of the Sudan. New York: Holt, Rinehart \& Winston.

Foot, P. [1958-59] (1978) 'Moral Beliefs', in her Virtues and Vices and Other Essays in Moral Philosophy. Oxford: Blackwell: 110-129.

Goldie, P. (2007) 'Towards a virtue theory of art', British Journal of Aesthetics 47: 372-87

Goldie, P. (2008) 'Virtues of art and human well-being', Proceedings of the Aristotelian Society Supp. Vol. LXXXII: 179-95.

Gomes, A. (2009) 'Goldie on the Virtues of Art', British Journal of Aesthetics 49: 75-81

Hawley, K. and Macpherson, F. (eds.) (2011) The Admissible Contents of Experience London: Blackwell

McDowell, J. [1979] (1998) 'Virtue and Reason', in his Mind, Value and Reality Cambridge MA: Harvard University Press: 50-73

30 This paper was written in 2010 whilst I was a member of the Department of Philosophy at Birkbeck College. My thanks to then-colleagues Miranda Fricker, Susan James and Anthony Price for conversations on these topics, and especial thanks to an anonymous referee whose comments have much improved the paper. 
McDowell, J. [1983] (1998) 'Aesthetic Value, Objectivity and the Fabric of the World', in his Mind, Value and Reality Cambridge MA: Harvard University Press: 112-130

McGinn, C. (1999) Ethics, Evil, and Fiction. Oxford: Oxford University Press

Moore, A.W. (2006) 'Maxims and Thick Ethical Concepts', Ratio XIX: 129-147

Murdoch, I. (1957) 'The Sublime and the Good'. Reprinted in her (Murdoch 1997).

Murdoch, I. (1959) 'The Sublime and Beautiful Revisited'. Reprinted in (Murdoch 1997).

Murdoch, I. (1992) Metaphysics as a Guide to Morals. London: Chatto \& Windus

Murdoch, I. (1997) Existentialists and Mystics: Writings on Philosophy and Literature, ed. P. Conradi. London: Chatto \& Windus.

Price, A.W. (2000) 'On Criticising Values', Royal Institute of Philosophy Supplement 47: 141-158

Phillips, K.C. (1970) Jane Austen's English. London: Andre Deutsch.

Putnam, H. (2002) The Collapse of the Fact/Value Dichotomy and other essays. Cambridge, MA: Harvard University Press

Roessler, J. (2005) 'Joint Attention and the Problem of Other Minds', in N. Eilan et.al. (eds.) Joint Attention: Communication and Other Minds. Oxford: Oxford University Press

Ryle, G. [1968] (2009) 'Jane Austen and the Moralists'. Reprinted in his Critical Essays: Collected Papers Volume 1. London: Routledge

Ryles, J. (1982) Warriors of the White Nile: The Dinka. Amsterdam: Time-Life

Siegel, S. (2005) 'Which Properties Are Represented in Perception?', in T. Szabo Gendler and J. Hawthorne (eds.) Perceptual Experience. Oxford: Oxford University Press

Weil, S. (2005) Simone Weil: An Anthology. S. Miles (ed.). London: Penguin.

Williams, B. (1985) Ethics and the Limits of Philosophy. London: Routledge

Wittgensetin, L. (1961) Tractatus Logico-Philosophicus, D.F. Pears and B.F. McGuinness (trans.). New York: Humanities Press. 